\title{
Synovial fluids facilitate small solute diffusivity
}

\author{
NORTIN M. HADLER \\ From the Departments of Medicine and Bacteriology (Immunology), University of North Carolina School of \\ Medicine, Chapel Hill, North Carolina, USA
}

SUMmaRY The diffusivity of water, fucose, proline, lysine, glutamic acid, glucose and sucrose was determined in a variety of inflammatory human synovial fluids. In view of the constituents of pathological synovial fluids one would predict impedence to solute movement. In several fluids diffusivity was enhanced relative to translational diffusion in water. In most fluids diffusivity was enhanced relative to diffusion in diluted serum. Diffusivity of the solutes was dependent on size and charge of the solute but independent of fluid characteristics, including glucose, protein, and complement concentrations and cellular constituents. This paper reports aberrant small solute behaviour in synovial fluids. Enhanced diffusivity relative to water was demonstrated in hyaluronate dissolved in diluted serum as well. Hyaluronate domains in the synovial fluids interact with these solutes, facilitating movement. This phenomenon affords a homoeostatic mechanism as regards chondrocyte viability in spite of inflammation or a low glucose concentration.

In the normal diarthrodial joint the subchondral bony plate is impermeable to small metabolites such as glucose and water. ${ }^{1}$ These small solutes must traverse the synovial fluid that is interposed between viable chondrocytes in cartilage and the vasculature of the synovium. Synovial fluid subserves an essential and obligate transport function central to the maintenance of normal articular physiology.

A small spherical solute with a radius $r$ moves through a simple liquid by the process of diffusion. This diffusional behaviour (D) is described by the Stokes-Einstein relation:

$$
\mathrm{D}=\frac{\mathrm{kT}}{6 \pi \mathrm{r} \eta}
$$

where $\mathbf{k}$ is the Boltzmann constant and $\mathrm{T}$ the absolute temperature. For spherical solutes in a simple solvent at a given $\mathrm{T}$ diffusivity is inversely dependent on the molecular radius and therefore on the molecular weight. For asymmetric solutes tumbling through the solvent the long axis becomes the effective or Stokes's radius. ${ }^{2}$

Synovial fluid is obviously not a simple solvent system. The presence of a polymeric network, the matrix of hyaluronic acid, influences the motion of solutes. Preston and Snowden ${ }^{3}$ have provided an synopsis of this complex interaction. The influence

Accepted for publication 27 November 1979

Correspondence to N. M. Hadler MD, Department of Medicine, University of North Carolina School of Medicine, Chapel Hill, N.C., 27514, U.S.A. of the matrix on solute diffusion can be described $\vec{\varphi}$ by a general relationship:

$$
\frac{\overline{\mathbf{D}}}{\overline{\mathrm{Do}}}=\frac{(\overline{\mathrm{A}} / \mathrm{Ao})(\overline{\mathrm{F}} / \mathrm{Fo})}{\mathrm{h}} \text {. }
$$

This equation defines the causal factors for any discrepancy in diffusion in the presence of the matrix ( $\bar{D})$ when compared to its absence (Do). $\overline{\mathrm{A}} / \mathrm{Ao}$ is the restriction in the total area available for diffusion, $\bar{F} / F o$ is a measure of the complex interactions between solute and matrix, including adsorptive phenomenon, solvent frictional drag, etc., and $\mathrm{h}$ is a measure of the tortuosity of the available paths for diffusion. In brief, a polymeric matrix can impede translational motion (from place to place) of a solute by occupying space and by interacting with the solute. This interaction can reflect weak binding of the solute to the matrix, or the matrix can restrict the tumbling motion of the solute. For example, in some polymeric matrices the diffusional behaviour of an asymmetric solute is dependent on the dimension of its short axis in contrast to its behaviour in a simple solvent. ${ }^{4}$ The presence of the polymer restricts the tumbling motion of the solute so that the solute tends to align parallel to the translational pathway.

If one examines the translational movement of sizeable solutes ( $>5000$ daltons) in synovial fluid, $\overline{\mathbf{D}} / \mathrm{Do}$ is less than unity. ${ }^{5}$ Such diminished relative diffusivity is a function of solute size as well as the concentration of hyaluronic acid. However, what are the mechanisms whereby small solutes move through 
the synovial fluid? This is an issue that has fascinated clinicians for decades. ${ }^{6}$ It is raised by the classic observation of exceedingly low glucose concentration in some rheumatoid synovial effusions. How do chondrocytes maintain viability bathed in a fluid that affords such a shallow concentration gradient for the translational movement of glucose? The studies reported here were undertaken to assess transport properties of pathological synovial fluids for small solutes by determining the bulk diffusion coefficients (D) for water, fucose, proline, lysine, glutamic acid, glucose, and sucrose.

\section{Materials and methods}

\section{DETERMINATION OF THE BULK}

\section{DIFFUSION COEFFICIENTS}

The methods employed are adopted from those utilised by Redwood et al. ${ }^{7}$ to determine red cell membrane permeability to water. At ambient temperature sufficient synovial fluid is aspirated into polyethylene tubing $0.086 \mathrm{~cm}$ in internal diameter or $0.039 \mathrm{~cm}$ internal diameter (Intramedic, Clay Adams, Inc., Parsippany, NJ) to produce a column of synovial fluid $5 \mathrm{~cm}$ in length. The radionuclide solution was applied in $0.5 \mu \mathrm{l}$ volume to one end of the fluid column in the $0.086 \mathrm{~cm}$ internal diameter tubing and in $0.1 \mu \mathrm{l}$ in the 0.039 tubing with a Hamilton syringe. Only for the experiment with tritiated water that end is sealed with clay. The tubing is fastened horizontally in a specially designed lucite block. After a timed incubation at $4^{\circ}$ or $37^{\circ} \mathrm{C}$ (water-saturated atmosphere) the block is placed at $-70^{\circ} \mathrm{C}$ for 30 minutes.

The lucite blocks are $7 \times 3 \times 1.8 \mathrm{~cm}$. Three parallel holes were drilled the length of the long axis $0.25 \mathrm{~cm}$ from the surface and $1 \mathrm{~cm}$ apart to accommodate the tubing. The blocks have machined grooves perpendicular to the axis of the tubing so that the frozen tubing can be sliced with a razor blade into $0.5 \mathrm{~cm}$ sections. Each section was placed in minivials (New England Nuclear, Boston, Mass.) in the presence of phase combining system (PCS) (Amersham Searle, Arlington Heights, Illinois) overnight before scintillation counting.

D was computed essentially as described by Redwood et al. ${ }^{7}$ One-dimensional semi-infinite diffusion through a homogeneous medium is assumed. The initial deposit of tracer, $\mathrm{m}_{0}(\mathrm{cpm} /$ $\mathrm{cm}^{2}$ ), at $\mathrm{x}=0$ and $\mathrm{t}=0$ is computed by summing the counts per minute (cpm) from the whole length of tubing. The theoretical solution for such diffusion is the equation

$$
\text { In } c=1 n m_{0}(\pi D t)^{-1 / 2}-x^{2} / 4 D t
$$

where $c\left(\mathrm{cpm} / \mathrm{cm}^{3}\right)$ is the concentration of solute at distance $x(\mathrm{~cm})$ and time $t$ (seconds). A semiloga- rithmic plot of $c$ against $\mathrm{x}^{2}$ is a straight line with the slope-1/4Dt from which $\mathrm{D}\left(\mathrm{cm}^{2} \mathrm{~s}^{-1}\right)$ is computed. For the experiments reported herein a program was written for the Hewlett Packard 9830A computer equipped with $\mathrm{x}-\mathrm{y}$ plotter and tape reader. Details of this program are available on request from the author. Data points and a regression line are plotted for each experiment and $\mathrm{D}$ calculated. The probability $\left(\mathbf{R}^{2}\right)$ of fit to the straight line was always $>0.95$ and in $95 \%$ of the experiments was $>0.98$.

\section{PREPARATION OF SOLUTES}

AND SOLVENTS

Tritiated water, D-glucose $\left(\mathrm{G}-{ }^{3} \mathrm{H}\right), \mathrm{L}-\left(4,5^{-3} \mathrm{H}(\mathrm{N})\right)-$ lysine, L-1-14 C-fucose, D, L-(1-14 C)-glutamic acid, $\mathrm{L}-\left({ }^{3} \mathrm{H}(\mathrm{G})\right)$-proline, and sucrose $\left({ }^{14} \mathrm{C}(\mathrm{U})\right)$ were purchased from New England Nuclear. With the exception of tritiated water and where specified, all solutes were dissolved in saline buffered at pH 7.0 with $0.01 \mathrm{M}$ sodium phosphate (PBS). The experiments presented were 'double-labelled' codiffusion experiments as detailed. Many experiments were repeated 'single-labelled' without influencing the $\mathbf{D}$ measured. The final concentration of the solutes ranged between 0.1 and $0.5 \mathrm{mM}$. The specific activity of the solute solution was adjusted such that $>3 \times 10^{4} \mathrm{cpm}$ was applied and the regression line fit to more than 7 points above background.

The inflammatory synovial fluids (SFs) utilised in these studies are catalogued in Table 1. In each case they were obtained from the knee of patients referred to the author's clinic, the arthrocentesis being part of the diagnostic evaluation. The fluids were clarified by centrifugation, aliquoted, and frozen at $-20^{\circ} \mathrm{C}$. Once thawed for use, the fluid was stored at $4^{\circ} \mathrm{C}$. No influence of freezing was demonstrable (SF1, SF5) when freshly obtained SF was compared to a thawed aliquot. Different aliquots of many of these fluids were thawed and studied on two occasions separated by more than a year. Differences in mean diffusivity were less than $10 \%$. The fluids are inflammatory with fair to poor 'mucin clots' ${ }^{8}$ SF3, 11, 13 are classical rheumatoid effusions in that they are exudative, decomplemented, and have markedly depressed glucose concentration. Radiographs taken in close proximity to the arthrocentesis were read by the author without reference to the diffusion data. They were scored $0-4(x$-ray score) for destruction as previously described. ${ }^{9}$

The water and serum solvent controls in $0.086 \mathrm{~cm}$, that is, tubing only, were prepared by methods similar to that of Redwood et al. ${ }^{7}$ in an agarose matrix with Indubiose A37 (Gallard, Schlesinger, NY). Horse serum was purchased from Gibco. 
Table 1 Description of the synovial fluids

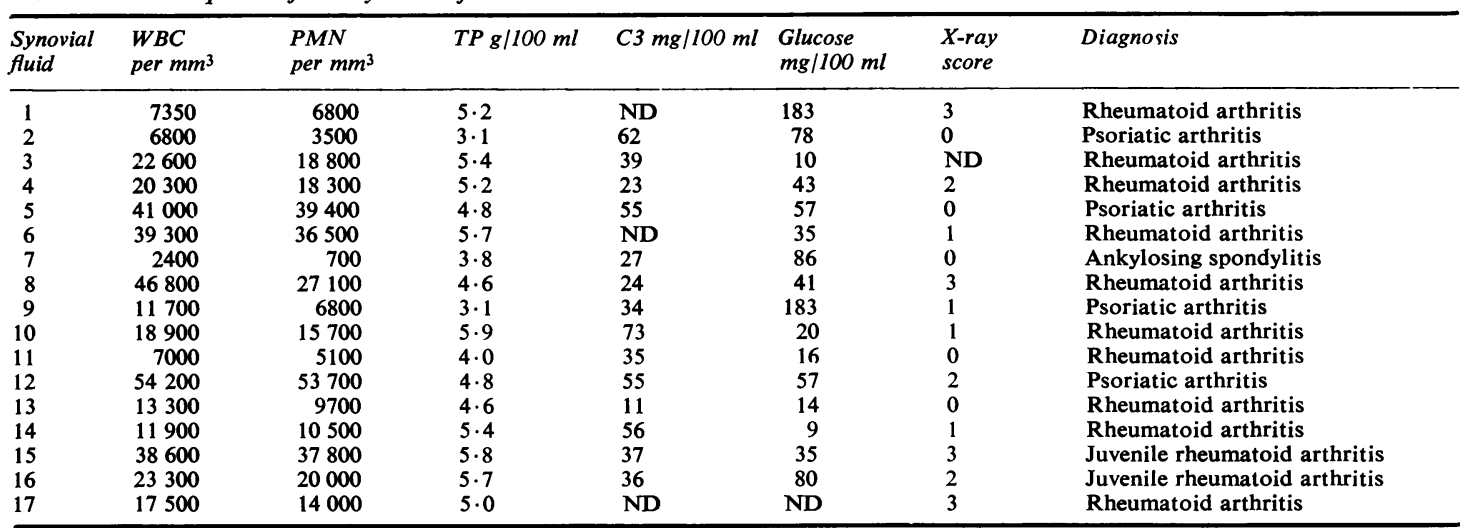

Abbreviations used include WBC for total leucocyte count, PMN for neutrophils, TP for total protein, and ND for not done. SI conversions: Number of leucocytes $\times 10^{9} / \mathrm{l}=$ number $/ \mathrm{mm}^{3} \times 10^{6} ;$ total protein $\mathrm{g} / \mathrm{l}=\mathrm{g} / 100 \mathrm{ml} \times 10 ; \mathrm{C3} \mathrm{g} / \mathrm{l}=\mathrm{mg} / 100 \mathrm{ml} \times 0.01 ; \mathrm{glucose} \mathrm{mmol} / \mathrm{l}=$ $\mathrm{mg} / 100 \mathrm{ml} \times 0.555$.

Hyaluronic acid extracted from human umbilical cord was purchased from Sigma Chemicals (H1876, St. Louis, Mo.).

\section{Results}

The bulk diffusion coefficients for tritiated water (Table 2) in water and serum are in reasonable agreement with previously reported values 71011 in spite of the fact that the current experiments were begun at ambient temperature and allowed to equilibrate during the measured time interval. Table 2 shows that synovial fluid as a solvent when compared to water alone as a solvent does not significantly retard the bulk diffusion of a tritiated water tracer. Furthermore, the movement of water is less impeded in synovial fluids, many of which are

Table 2 Diffusion coefficients for tritiated water $\left(10^{-5} \mathrm{~cm}^{2} \mathrm{sec}^{-1}\right)^{*}$

\begin{tabular}{|c|c|c|c|c|}
\hline \multirow{2}{*}{$\begin{array}{l}\text { Temperature } \\
\text { Tubing ID }(\mathrm{cm}) \\
\text { Test solvent } \dagger\end{array}$} & \multicolumn{2}{|l|}{$4^{\circ} \mathrm{C}$} & \multicolumn{2}{|l|}{$37^{\circ} \mathrm{C}$} \\
\hline & 0.039 & 0.086 & 0.039 & 0.086 \\
\hline $\begin{array}{l}\mathrm{H}_{2} \mathrm{O} \\
50 \% \text { serum } \\
0.5 \% \text { hyaluronate } \\
\text { SFs }\end{array}$ & $\begin{array}{l}1 \cdot 80 \\
1 \cdot 59 \\
1 \cdot 89 \\
1 \cdot 71\end{array}$ & $\begin{array}{l}1 \cdot 88 \\
1 \cdot 64 \\
1 \cdot 91 \\
1 \cdot 71\end{array}$ & $\begin{array}{l}3 \cdot 94 \\
3 \cdot 44 \\
3 \cdot 85 \\
3 \cdot 82\end{array}$ & $\begin{array}{l}3 \cdot 99 \\
3 \cdot 59 \\
3 \cdot 85 \\
3 \cdot 81\end{array}$ \\
\hline
\end{tabular}

*Data are presented as the mean of replicate experiments. For $\mathrm{H}_{2} \mathrm{O}$, $50 \%$ serum, and hyaluronate 6 observations were made for each variable. Ten synovial fluids (SFs) were studied in triplicate at least. The mean was computed for replicate experiments. The data presented in this table as 'SFs' are the mean of the values determined for each SF. The SEM for all these experiments averaged $7 \%$ of the respective mean value.

†As described in the Methods section, $\mathrm{H}_{2} \mathrm{O}$ and $50 \%$ serum solvents in the $0.086 \mathrm{~cm}$ tubing were prepared with an agarose matrix. No agarose was included when these solvents were prepared in the $0.039 \mathrm{~cm}$ tubing.

$\mathbf{W}=$ internal diameter.

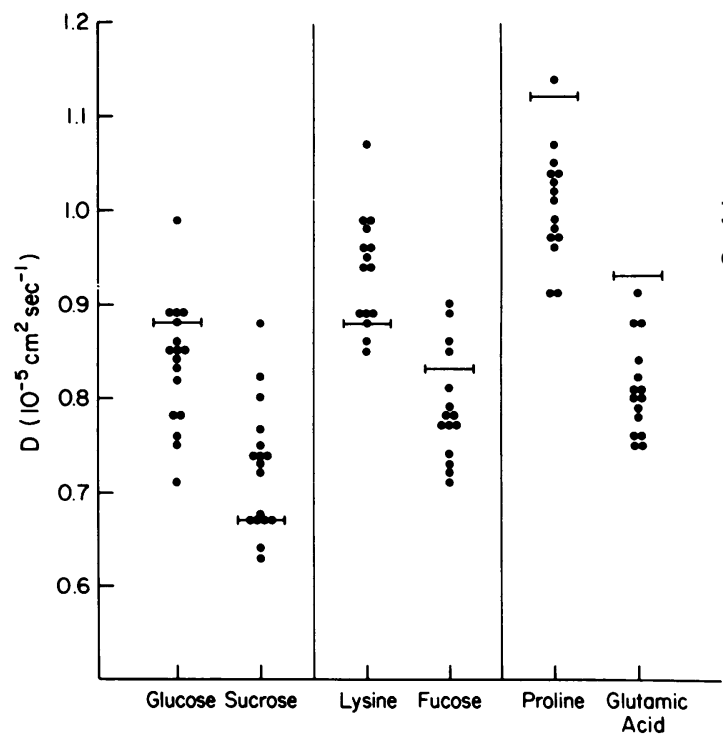

Fig. 1 Apparent bulk diffusion coefficients $(D)$ for the indicated amino acids and sugars in the individual synovial fluids (Table 1). Each point is the mean of a minimum of triplicate experiments. The standard error of the mean for all these experiments averaged $6 \%$ of the respective mean. All experiments are codiffusion experiments which paired the solutes as illustrated. Of each pair the solute to the left was employed as a tritiated radionuclide and that to the right was ${ }^{14} \mathrm{C}$ labelled. The line is the mean $D$ for the particular solute diffusing in agarose dissolved in PBS (Table 3-II).

When contrasted with diffusion in agarose-PBS some $S F$ appear to enhance the translational movement of some of the solutes, particularly sucrose and lysine. However, in contrast to diffusion in agarose- $50 \%$ serum (Table 3-II), most of these exudative SFS enhance translational movement of all the solutes. 
exudative (Table 1), than in $50 \%$ human serum. Convection is unlikely to have contributed significantly to these results in view of the temperaturedependent viscoelastic properties of SF, because the relative diffusivity $\left(\mathrm{SF}: \mathrm{H}_{2} \mathrm{O}\right)$ pertains at both $4^{\circ}$ and $37^{\circ} \mathrm{C}$. In our hands, as in the experiments of Redwood et al., ${ }^{7}$ the $\mathrm{D}$ for tritiated water in the $0.086 \mathrm{~cm}$ tubing with agarose is indistinguishable from that in the $0.039 \mathrm{~cm}$ tubing without agarose. This narrower tubing by virtue of its geometry minimises the influence of convection even for water as a solvent. Were convection operating in the synovial fluids, one would predict a discrepancy between $\mathrm{D}$ measured in 0.086 and $0.039 \mathrm{~cm}$ internal diameter tubing. No significant discrepancy was detected.

Fig. 1 displays the results of codiffusion experiments for six solutes utilising the SFs (described in Table 1) as solvents. When compared with diffusivity of the particular solute in water, some SFs provide a solvent system which enhances translational movement. This phenomenon is particularly striking for sucrose and lysine. However, all these SFs are exudative, containing considerable quantities of protein (Table 1). Therefore a more appropriate comparison is to contrast solute movement in SF with that in dilute serum. The diffusion experiments (Table 3-II) in agarose dissolved in PBS or $50 \%$ serum permit such a comparison. ${ }^{712}$ Diffusivity of all the solutes in most SFs (Fig. 1) is strikingly enhanced when compared to their diffusivity in agarose dissolved in $50 \%$ serum (Table 3-II).
Table 3-I also presents data assessing small solute diffusivity in solvents in which matrices of hyaluronate are incorporated. When dissolved in PBS in a concentration of $0.5 \%$ or $1.0 \%$, hyaluronate does not greatly influence the diffusion of the solutes studied when contrasted with their translational movement in an agarose matrix. However, when serum proteins are included in the hyaluronate matrix, there is striking enhancement of translational movement of all the solutes. This is contrary to predicted solute behaviour as exemplified by the experiments in agarose. The incorporation of serum proteins in an agarose matrix impedes translational movement of these solutes.

Central to the interpretation of these data is the contention that convection is not contributing significantly to the observed solute movement. It is therefore useful to re-emphasise the data on which this contention is based. The diffusivity of tritiated water in SF in $0.086 \mathrm{~cm}$ internal diameter tubing is indistinguishable from that in $0.039 \mathrm{~cm}$ internal diameter tubing wherein convection, even in water without agarose, is insignificant ${ }^{7}$ (Table 2). The enhanced diffusivity is apparent at low temperatures when the increased viscosity of SF should impede convection (Table 2). The ability to discriminate the Ds for glucose and sucrose in codiffusion experiments (Fig. 1) likewise would be impaired were convection operating. In these codiffusion experiments the difference between glucose and sucrose in each test solvent is highly uniform and similar in magnitude to previously reported

Table 3 Influence of matrix, solvent, and solute diluent on $D\left(10^{5} \mathrm{~cm}^{2} \mathrm{sec}^{-1}\right)^{*}$

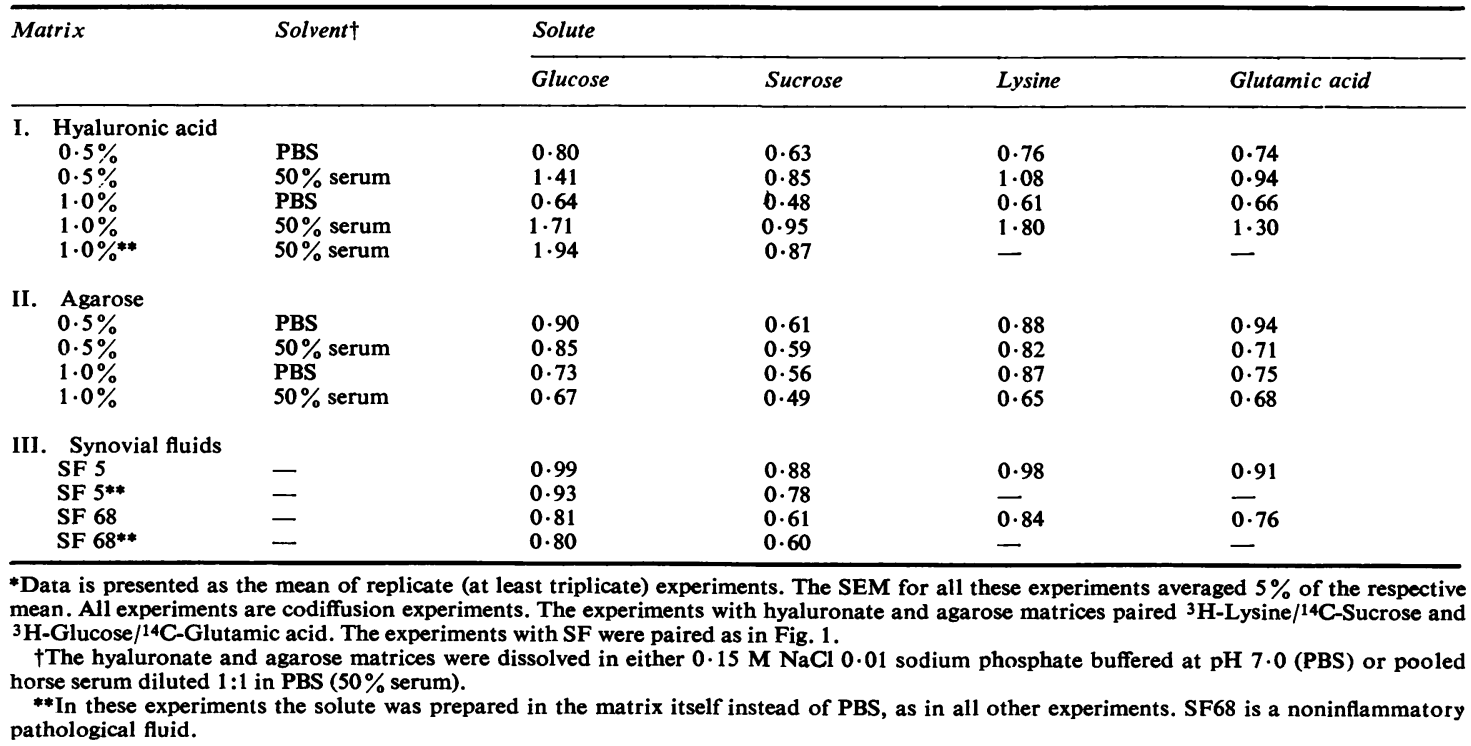


values. ${ }^{12}$ Finally, an interface is established, albeit very transiently, between the solute applied in a droplet of PBS and the solvent column studied. It is conceivable that the dissipation of this interface influences the initiation of translational movement by the solute and therefore the measurement of D. For this reason the results of several control experiments are presented in Table 3. Dissolving the radiolabelled solute in the same solvent as that in which $\mathrm{D}$ was measured, thereby avoiding any interface, does not alter the result.

Although the synovial fluids studied are all inflammatory and sampled from the knees of patients with erosive synovitides, there is a considerable range of constituent and $x$-ray score (Table 1). A correlation analysis ${ }^{9}$ was performed for each of the quantitative variables in Table 1 and the diffusion coefficients for each of the solutes in Fig. 1. There is no significant relationship $(P>0.05)$.

\section{Discussion}

This study shows that the apparent diffusivity of several sugars and amino acids within some pathological human synovial fluids is not depressed relative to that in water. Relative to a diluted serum solvent apparent diffusivity is often enhanced. Such anomalous solute behaviour has not previously been appreciated in a biological fluid. It is particularly surprising in pathological synovial fluids.

In a recent revew ${ }^{5}$ I discussed the structure of hyaluronic acid in synovial fluid and its influence on the movement of solutes. Hyaluronic acid is an unbranched polysaccharide consisting of regularly alternating glucosamine and glucuronate. Although polydisperse, its average molecular weight in normal human synovial fluid is $6 \times 10^{6}$ daltons and its concentration ranges between 0.14 and $0.36 \%$ of the net weight. In solution the hyaluronate chain is folded into a solvated spherical molecule or domain with a diameter exceeding 200 millimicrons. From these considerations and the known range of hyaluronate concentrations in synovial fluids it is clear that the dynamic, hydrated domains greatly overlap and interact, resulting in concentration-dependent, non-Newtonian viscoelastic properties of these fluids.

This 3-dimensional continuous chain network imparts to the fluid the properties of a molecular sieve, ${ }^{13} 14$ The greater the molecular dimensions of the solute, the more impeded its translational movement. For human albumin rotational freedom is retained in a hyaluronate matrix although translational movement is impeded. ${ }^{15}$ One corollary of the reported diminished translational diffusivity is the concept of an 'exclusion volume'. ${ }^{16-18}$ Pro- teins are excluded from the interstices of the hyaluronate matrix, and this exclusion is directly dependent both on the volume and shape of the protein molecule and on the hyaluronate concentration. At the hyaluronate concentration of normal synovial fluid albumin is excluded from $1 / 3$ of the total volume. Therefore when one measures the concentration of any protein constituent in synovial fluid, the value is an averaging for a highly nonhomogeneous solution. At any given region of the solution at any instant there is a probability that the solute will be sterically excluded by the matrix.

In view of these considerations of SF structure and composition, the D I have measured for the small solutes is not truly a bulk diffusion coefficient, as defined in the Methods section, since the solvent SFs are not homogeneous. However, all considerations of SF structure would suggest that the relative diffusivity should be depressed and not enhanced as observed: translational motion through the proteincontaining exclusion volume should be retarded. This is demonstrated in Table 2, where the diffusivity of water in $50 \%$ serum in significantly depressed, confirming the observation of Redwood et al., ${ }^{7}$ and in Table 3 for several of the other solutes. Therefore the data are consistent with the postulate that continuous 'channels' for movement of these small solutes exist in the interstices of the domains where larger proteins are absent. The observation that hyaluronate itself in concentrations similar to that in SFs (Table 3) can enhance relative diffusivity when dissolved in diluted serum lends credence to this postulate.

However, it is inadequate to visualise the 'channels' as holes or pores, since that would lead one to predict diminished diffusivity. Such pores would decrease the effective cross-sectional area of the column of SF. The observed enhanced solute movement implies more than channels but an interaction between the solute and the hyaluronate domains that facilitates movement. We have recently demonstrated enhanced solute diffusivity in concentrated $(2.5 \%)$ hyaluronate matrix without the addition of serum. Perturbing the inherent order of such an isolated dense hyaluronate matrix with calcium will abrogate the enhanced diffusivity of small solutes. ${ }^{19}$ These experiments are consistent with a direct interaction between the small solute and the matrix underlying translational movement.

The results of the current study differ from those reported by Ogston and Sherman..$^{13} \mathrm{I}$ have measured solute movement within SF. Those authors measured interfacial movement, i.e., the rate of transport (mass/area/time) of glucose from an aqueous compartment containing hyaluronate across a membrane to a compartment lacking hyaluronate. 
Such transport (out of the hyaluronate domains and through a membrane) was markedly depressed by increasing concentrations of hyaluronate. Preston and Snowden ${ }^{3}$ measured small solute movement within an isolated hyaluronate matrix and found, as I do, no restriction in translational movement.

Experiments further examining the behaviour of small solutes in the interstices of the dynamic domains of the various glycosaminoglycans are in progress. The data presented herein suggest that such domains interact with small solutes facilitating their movement in synovial fluid and perhaps in relatively avascular connective tissue elsewhere. Furthermore these domains may afford enhanced solute transport even in the setting of inflammation. Rheumatoid synovial effusions are exudative and often have a low glucose concentration (Table 1). The depressed glucose concentration relative to serum has been attributed to impaired transport through the synovium, ${ }^{6}$ though in the normal knee glucose diffusivity across the synovium is enhanced compared to other solutes..$^{20}$ The aberrantly rapid translational movement of some small metabolities such as glucose in the synovial fluid may afford a homoeostatic mechanism as regards chondrocyte viability in spite of a low glucose concentration.

Dr Hadler is the recipient of an Established Investigatorship from the American Heart Association. This work was supported in part by Research Grant AM 19992 from the National Institutes of Health, USPHS.

I thank Dr J. Parker for many stimulating discussions and for suggesting several methodological improvements, Dr A. Finn for a critical review of the manuscript, Ms $\mathrm{K}$. Andrews and Ms S. Chung for technical assistance, Ms Margaret Honeycutt for secretarial assistance, and Dr M. Dillard for assistance with data analysis.

\section{References}

1 Maroudas A, Bullough P, Swanson S A V, Freeman M A R. The permeability of articular cartilage. $J$ Bone Joint Surg 1968; 50B: 166-77.

2 Tanford C. Physical Chemistry of Macromolecules. New York: Wiley, 1961.

${ }^{3}$ Preston B N, Snowden J M. In: eds. Kukonen E, Pikkarainen J, Biology of the Fibroblast, New York: Academic Press, 1973: 215-30.
4 Lieb W R, Stein W D. Implications of two different types of diffusion for biological membranes. Nature New Biol 1971; 234: 220-2.

5 Hadler N M, Napier M A. The structure of hyaluronic acid in synovial fluid and its influence on the movement of solutes. Semin Arthritis Rheumat 1977; 7: 141-52.

6 Ropes M W, Muller A F, Bauer W. The entrance of glucose and other sugars into joints. Arthritis Rheum 1960; 3: 496-514.

7 Redwood W R, Rall E, Perl W. Red cell membrane permeability deduced from bulk diffusion coefficients. J Gen Physiol 1974; 64: 706-29.

8 Cohen A S, Brandt K D, Krey P R. In: Cohen A S, ed. Laboratory Diagnostic Procedures in the Rheumatic Diseases. Boston: Little Brown, 1975; 1-64.

9 Hadler N M, Spitznagel J K, Quinet R J. Lysosomal enzymes in inflammatory synovial effusions. J Immunol 1979; 123: 572-7.

10 Wang $J$ H. Self-diffusion and structure of liquid water. III. Measurement of the self-diffusion of liquid water with ${ }^{2} \mathrm{H},{ }^{3} \mathrm{H}$, and ${ }^{18} \mathrm{O}$ as tracers. J Am Chem Soc 1953; 75: 466-70.

11 Gary-Bobo C M, Weber H W. Diffusion of alcohols and amides in water from 4 to $37^{\circ}$. J Phys Chem 1969; 73: 1155-6.

12 Schantz E J, Lauffer M A. Diffusion measurements in agar gel. Biochemistry 1962; 1: 658-63.

13 Ogston A G, Sherman T F. Effects of hyaluronic acid upon diffusion of solutes and flow of solvent. $J$ Physiol 1961; 156: 67-74.

14 Laurent T C, Bjork I, Pietruszkiewicz A, Persson H. On the interaction between polysaccharides and other macromolecules. II. The transport of globular particles through hyaluronic acid solutions. Biochim Biophys Acta 1963; 78: 351-9.

15 Preston B N, Obrink B, Laurent T C. The rotational diffusion of albumin in solutions of connective-tissue polysaccharides. Eur J Biochem 1973; 33: 401-6.

16 Ogston A G, Phelps C F. The partition of solutes between buffer solutions and solutions containing hyaluronic acid. Biochem $J$ 1960; 78: 827-33.

17 Laurent T C. The interaction between polysaccharides and other macromolecules. 9. The exclusion of molecules from hyaluronic acid gels and solutions. Biochem $J$ 1964; 93: 106-12.

18 Laurent T C. In: Balazs E A, ed. Chemistry and Molecular Biology of the Intercellular Matrix. New York: Academic Press, 1970: 703-32.

19 Napier M A, Hadler N M. The effect of calcium on structure and function of a hyaluronic acid matrix: C-13 nuclear magnetic resonance analysis and the diffusional behaviour of small solutes. Proc Nat Acad Sci USA 1978; 75: 2261-5.

20 Simkin P A, Pizzorno J E. Transynovial exchange of small molecules in normal human subjects. $J$ Appl Physiol 1974; 36: 581-7. 